\title{
Minimally Invasive Video-Assisted Parathyroidectomy; A Detailed Illustration and Highlighting an Additional Potential Advantage Over Other Targeted Parathyroid Surgeries
}

Sohail Bakkar*, Marco Biricotti, Lorenzo Fregoli, Valeria Matteucci, Piermarco Papini, Salvatore Pagliaro, Gianluca Frustaci, Aleksandr Aghababyan, Carlo Enrico Ambrosini, David Galleri, Gabriele Materazzi, and Paolo Miccoli

Department of Surgical Pathology, University Hospital of Pisa, Italy

\begin{abstract}
Targeted parathyroid surgery also referred to as minimally invasive parathyroidectomy has replaced full neck exploration as the preferred surgical approach to primary hyperparathyroidism. This is attributed to the ability to accurately localize enlarged parathyroid glands preoperatively and obtain objective evidence of adequate resection intraoperatively. The two most widely used minimally invasive parathyroid surgeries are the non-endoscopic miniincision parathyroidectomy and the minimally invasive video-assisted parathyroidectomy. The aim of this article is to provide a detailed illustration of the latter supplemented with an animated video of the procedure, and to highlight a potential advantage it offers over other targeted parathyroid procedures; the ability to perform a full neck exploration and/or a concomitant thyroid surgery without the need to convert to a standard cervicotomy.
\end{abstract}

Keywords: Minimally invasive; Video-assisted; Targeted; Focused; Parathyroidectomy

\section{Introduction}

Surgery is the only curative treatment modality for primary hyperparathyroidism (PHP), and full neck exploration was considered the gold standard approach. However, because the majority of cases of PHP are due to single gland disease and because of the ability to accurately localize enlarged parathyroid glands preoperatively, and obtain objective evidence of adequate resection intraoperatively; focused parathyroid surgery (also known as minimally invasive parathyroid surgery) has replaced full neck exploration as the preferred surgical strategy. Minimally invasive parathyroidectomy is comparable to its conventional counterpart in terms of safety and cure rate. However, it offers the additional advantages of reduced operative time, hospital stay, and cost, early biochemical and symptomatic hypocalcemia, and improved cosmetic and pain outcomes [1-3]. The two most widely used targeted parathyroid surgeries are the non-endoscopic miniincision parathyroidectomy (MIP) and the minimally-invasive videoassisted parathyroidectomy (MIVAP). A large retrospective casecontrolled study demonstrated that both procedures were equally safe and effective. However, pain and cosmetic outcomes were in favor of MIVAP [4]. This article provides a detailed description of MIVAP's technique and is supplemented with a video demonstration of the procedure. It also highlights an additional advantage of MIVAP over other minimally invasive parathyroid procedures $[5,6]$.

\section{Procedural steps}

i. Access.

ii. Identification of the abnormal parathyroid gland.

iii. Identification of the recurrent laryngeal nerve (RLN).

iv. Excision of the abnormal gland.

v. Closure.

\section{General settings and access}

Patient's position: The patient is placed supine on the operating table with the arms tucked at the sides. The neck should be midline and only slightly extended. Extending the neck as in conventional surgery reduces the working space. The lack of neck extension offers an additional advantage; it minimizes the degree of potential postoperative neck discomfort

Anesthesia: Local-regional anesthesia and general anesthesia with endotracheal intubation are both viable options. In the authors' practice the procedure is performed under general anaesthesia with local anesthesia being reserved for the rare situation in which the patients' comorbidties favor avoiding general anesthesia.

Parathyroid hormone (PTH) level measurement: Prior to commencing the procedure a basal serum PTH measurement is obtained in the theater.

Site preparation: The skin is prepped from the lower lip to the nipples.

Position of the surgical team: The surgical team consists of three surgeons. The operator always stands on the patient's right side regardless of the location of the pathology. The first assistant stands on the patient's left side and is in charge of holding the camera. The second assistant stands at the head of the operating table above the patient's head, holding the retractors.

Instruments: The set of instruments used in MIVAP could be assembled from different sets of instruments used in General Surgery and Otorhinolaryngology. No Energy devices are required. However, ultrasonic shears can be used if the need for a concomitant thyroid lobectomy emerges.

*Corresponding author: Sohail Bakkar MS, MD, Division of Endocrine Surgery, Department of Surgical Pathology, University Hospital of Pisa, Via Paradisa2, 56124 Pisa, Italy, Tel: +393441162487; Fax: +39050997709; E-mail: sohail.bakkar@gmail.com

Received August 18, 2016; Accepted September 19, 2016; Published September 26, 2016

Citation: Bakkar S, Biricotti M, Fregoli L, Matteucci V, Papini P, et al. (2016) Minimally Invasive Video-Assisted Parathyroidectomy; A Detailed Illustration and Highlighting an Additional Potential Advantage Over Other Targeted Parathyroid Surgeries. Surgery Curr Res 6: 278. doi: 10.4172/2161-1076.1000278

Copyright: ( 2016 Bakkar S, et al. This is an open-access article distributed under the terms of the Creative Commons Attribution License, which permits unrestricted use, distribution, and reproduction in any medium, provided the original author and source are credited. 
Citation: Bakkar S, Biricotti M, Fregoli L, Matteucci V, Papini P, et al. (2016) Minimally Invasive Video-Assisted Parathyroidectomy; A Detailed Illustration and Highlighting an Additional Potential Advantage Over Other Targeted Parathyroid Surgeries. Surgery Curr Res 6: 278. doi: 10.4172/2161-1076.1000278

Page 2 of 3

\section{Executional steps}

A $1.5 \mathrm{~cm}$ transverse incision is made two finger breadths above the sternal notch disguised in a neck crease if possible. The incision should never be higher than the cricoid cartilage. The incision is deepened through the subcutaneous fat and platysma using electrocautery. Subplatysmal flaps are not raised. The cervical linea Alba is then exposed by lateral retraction using small retractors. It is then incised vertically using electrocautery and the thyroid gland is exposed. The thyroid gland is then bluntly dissected from the overlying strap muscles by gentle traction and the use of a tiny spatula. Once the thyroid gland has been completely dissected free from the strap muscles, the small retractors are replaced by two larger ones that are used to maintain the working space throughout the procedure. The thyroid lobe is placed under the medial retractor and the lateral one is used to retract the strap muscles laterally. A $5 \mathrm{~mm} 30^{\circ}$ camera is then introduced and preparation of the working space is completed using two spatulas. Typically, the camera is held at a $90^{\circ}$ angle on the field with its $30^{\circ}$ tips looking upward. The abnormal gland is identified. Grossly, an adenomatous parathyroid gland is large and tan or beefy red. Once identified, excision should not be attempted until the RLN is identified and preserved. The abnormal parathyroid gland is then grasped from the adipose tissue attached to it using a special grasper. Care must be taken not to grasp it directly from its capsule due to the potential risk of capsule rupture and subsequent parathyromatosis. The gland is then bluntly dissected from its surroundings using a spatula. This helps expose its vascular pedicle. The pedicle is then controlled using small metallic clips and subsequently divided, and the parathyroid gland is removed. An appropriate drop in serum PTH levels should be confirmed by obtaining an intraoperative measurement five minutes following pedicle ligation.

Finally, the surgical site is carefully inspected for adequate hemostasis. No drains are placed. The muscles are reapproximated with a single figure-of-eight suture using 3-0 Vicryl. The subcutaneous tissue is approximated by two simple interrupted sutures using 4-0 Vicryl and the skin is closed with glue. The procedure is demonstrated in the provided animated-video (Video). Its contraindications are broadly classified into absolute and relative and are demonstrated in Table 1.

\section{Comments}

At times, the need for a bilateral exploration and/or performing a concomitant thyroid lobectomy or even a total thyroidectomy emerges.

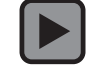

\begin{tabular}{|c|c|}
\hline Absolute & Relative \\
\hline Thyroid volume $>30 \mathrm{ml}$ & Lack of pre-operative localization \\
\hline Adenomas $>4 \mathrm{~cm}$ & Four-gland disease (hyperplasia) \\
\hline Previous neck surgery & Suspicion of a parathyroid carcinoma \\
\hline
\end{tabular}

*The authors' consider a parathyroid lesion clinically suspicious of a carcinoma a relative contraindication. Surgeons with adequate proficiency in performing minimally invasive video-assisted thyroidectomy (MIVAT) could perform an en bloc parathyroidectomy and thyroid lobectomy via the same access without the need to convert to a standard cervicotomy $[5,6]$

Table 1: Contraindications to minimally invasive video-assisted parathyroidectomy (MIVAP).

Double adenomas, intrathyroidal parathyroid lesions and lesions suspicious of carcinomas are all rare but well recognized entities [79]. This represents a limitation to most minimally invasive parathyroid surgeries described in literature such as MIP, and the video-assisted lateral approach [10]. On the other hand, MIVAP in experienced hands allows for both conducting a full neck exploration and for performing a concomitant thyroid surgery without the need to convert to a standard cervicotomy [5,11]. This is attributed to its central and direct access, in addition to the adequacy of the working space created by the procedure. The working space in MIVAP is identical to that created for minimally invasive video-assisted thyroidectomy [6]. This property of MIVAP allowed for demonstrating its feasibility in the management of four-gland disease [12]. Accordingly, four-gland disease (hyperplasia), lesions clinically suspicious of a parathyroid carcinoma, and the lack of pre-operative localization should no longer be considered absolute contraindications to MIVAP when sufficient experience exists (Table 1).

A 5-year experience with MIVAP at the authors' center has been reported in literature [13]. The outcomes of 260 procedures were reported, and potential limitations and drawbacks were concluded. The authors' experience with MIVAP has been ever-growing. In the past year 77 procedures were performed with a mean operative time of $31 \pm 20.4$ minutes, and an incision of a mean length of $17 \mathrm{~mm}$. None of the patients developed vocal fold palsy nor experienced postoperative bleeding requiring revision surgery. The mean visual analogue scale (VAS) score for the patients was 2, and all patients were cured following the first operation. One patient required a concomitant thyroid lobectomy due to features suspicious of a parathyroid carcinoma, and exploration was required in two patients because of the normality of the glands on the side suggested by imaging studies. In none of these three patients was conversion to standard cervicotomy required.

\section{Conclusion}

The usefulness of MIVAP not only lies in its ability to offer the advantages of minimally invasive procedures, but also in its ability to overcome the limitations of such procedures. Unlike other targeted parathyroid procedures it allows full neck exploration and/or performing an en bloc thyroid lobectomy without the need to convert to a standard cervicotomy. This makes it an appealing procedure and an integral part of the modern Endocrine Surgeon's armamentarium.

\section{Disclosure}

The authors have no financial ties or conflicts of interest to disclose.

\section{References}

1. Miccoli P, Bendinelli C, Berti P, Vignali E, Pinchera A, et al. (1999) Video-assisted versus conventional parathyroidectomy in primary hyperparathyroidism: a prospective randomized study. Surgery126: 1117-1121.

2. Udelsman R (2002) Six hundred fifty-six consecutive explorations for primary hyperparathyroidism. Ann Surg 235: 665-670.

3. Bergenfelz A, Kanngiesser V, Zielke A, Nies C, Rothmund M

$(2005$ 
Citation: Bakkar S, Biricotti M, Fregoli L, Matteucci V, Papini P, et al. (2016) Minimally Invasive Video-Assisted Parathyroidectomy; A Detailed Illustration and Highlighting an Additional Potential Advantage Over Other Targeted Parathyroid Surgeries. Surgery Curr Res 6: 278. doi: 10.4172/2161-1076.1000278

Conventional bilateral cervical exploration versus open minimally invasive parathyroidectomy under local anaesthesia for primary hyperparathyroidism. Br J Surg 92: 190-197.

4. Barczyński M, Papier A, Kenig J, Nawrot I (2014) A retrospective case-controlled study of video-assisted versus open minimally invasive parathyroidectomy. Videosurgery and other Miniinvasive Techniques 9: 537-547.

5. Miccoli P, Materazzi G, Bonari G, Donatini G, Berti P (2008) Minimally-invasive video-assisted parathyroidectomy. Operative techniques in Otolaryngology 19 : 22-25.

6. Bakkar S, Materazzi G, Biricotti M, De Napoli L, Conte M, et al. (2016) Minimally Invasive Video-Assisted Thyroidectomy (MIVAT) From A to Z. Surg Today 46: 255-259.

7. Tezelman S, Shen W, Shaver JK, Siperstein AE, Duh QY, et al. (1993) Double parathyroid adenomas. Clinical and biochemical characteristics before and after parathyroidectomy. Ann Surg218: 300-309.

8. Goodman A, Politz D, Lopez J, Norman J (2011) Intrathyroid parathyroid adenoma: incidence and location--the case against thyroid lobectomy. Otolaryngol Head Neck Surg 144: 867-871.

9. Wei $\mathrm{CH}$, Harari A (2012) Parathyroid carcinoma: update and guidelines for management. Curr Treat Options Oncol 13: 11-23.

10. Henry JF, Defechereux T, Gramatica L, de Boissezon C (1999) Minimally invasive videoscopic parathyroidectomy by lateral approach. Langenbecks Arch Surg 384: 298-301

11. Alesina PF, Singaporewalla RM, Walz MK (2010) Video-assisted bilateral neck exploration in patients with primary hyperparathyroidism and failed localization studies. World J Surg 34: 2344-2349.

12. Alesina PF, Hinrichs J, Kribben A, Walz MK (2010) Minimally invasive videoassisted parathyroidectomy (MIVAP) for secondary hyperparathyroidism report of initial experience. Am J Surg 199: 851-855.

13. Berti P, Materazzi G, Picone A, Miccoli P (2003) Limits and drawbacks of videoassisted parathyroidectomy. Br J Surg 90: 743-747. 